\title{
Laparotomía, laparoscopia diagnóstica, laparoscopia operatoria: análisis de costos
}

\author{
Ramiro Argüello Argüello*
}

\section{RESUMEN}

Se analizan los costos en un caso representativo de Dolor Pélvico Crónico, Se demuestra cómo la práctica de la laparoscopia diagnóstica, sin la posibilidad de un procedimiento laparoscópico operatorio en el mismo acto quirúrgico, puede llevar a una laparotomía en un tiempo quirúrgico posterior. En Colombia esto puede causar aumentos en el costo de la atención de la paciente entre un 97 a $124 \%$.

PALABRAS CLAVES: Costos, laparoscopia, laparotomía, dolor pélvico cróuico, Colombia.

\section{SUMMARY}

Costs involved in a representative case of a Chronic Pelvic Pain is analized. The practice of Diagnostic Laparoscopy, without the possibility of Operative Laparoscopy in the same surgical procedure could lead to a laparotomy in another delayed surgical time. In Colombia this might cause higher costs in patient's care in about 97 to $124 \%$.

KEY WORDS: Cost studies, laparoscopy, laparotomy, chronic pelvic pain, Colombia.

\section{Introducción}

En un artículo anterior analizamos los costos comparativos entre Laparoscopia Ginecológica Ambulatoria y la cirugía ginecológica por Laparotomía. Allí se demostró que cuando se tiene en cuenta el costo generado por la incapacidad de la paciente, es mejor realizar cirugía por laparoscopia y no por laparotomía (1).

Se debe tener en cuenta la enorme difusión y acogida que tienen los procedimientos laparoscópicos en el mundo, especialmente en ginecología (2-3). En este sentido los ginecólogos de Colombia siguen esa tendencia (4-5), y cada vez es más frecuente la presión por parte de las pacientes para que su cirugía se realice por vía laparoscópica (3), evitando la cicatriz de la laparotomía.

Se debe recordar que hoy día un gran número de los procedimientos que anteriormente se debían realizar mediante laparotomía se hacen ahora, preferiblemente, de manera ambulatoria por videolaparoscopia (6-7), y que actualmente las LAPAROSCOPIAS GINECOLOGICAS OPERATORIAS se encuentran incluidas en los manuales de tarifas del Instituto de Seguros Sociales

*Coordinador de la Comisión de Certificación en Endoscopia Ginecológica de la Sociedad Colombiana de Obstetricia y Ginecología. Certificación de Experto en Endoscopía Ginecológica dado por la Sociedad Colombiana de Obstetricia y Ginecología. Director Científico de la Unidad de Endoscopia Ginecológica y Dolor Pélvico. UNEGIM LTDA.
(1. S.S.) y del Seguro Obligatorio de Accidentes de Tránsito (S.O.A.T.). Los diferentes Niveles de Cirugía Laparoscópica contemplados en estos Manuales de Tarifas ya cuentan con más del $60 \%$ a $70 \%$ de los procedimientos que anteriormente solo se hacían mediante Laparotomía (8-9). Es muy importante resaltar que al efectuar estas cirugías mediante Laparoscopia se están convirtiendo, en su mayoría, en CIRUGIAS AMBULATORIAS, con ventajas innegables para las pacientes (6), permitiendo al mismo tiempo un mejor manejo de las camas hospitalarias en las instituciones.

Como la frecuencia y presión para realizar cirugía ginecológica por laparoscopia en lugar de disminuir parecen ir en aumento, merece la pena analizar qué pasa en Colombia, en materia de costos, cuando una determinada E.P.S. u otro organismo de salud no acepta este cambio, que ya es mundial, y rechaza la posibilidad de realizar cirugía ginecológica por laparoscopia.

\section{Materiales y métodos}

La Unidad de Endoscopia Ginecológica y Dolor Pélvico, UNEGIN, dirige desde hace siete años programas de cirugía ginecológica laparoscópica en instituciones de II y III nivel. Hemos tenido la oportunidad de trabajar con la mayoría de las Empresas Promotoras de Salud (E.P.S.) de nuestro medio, y gracias a esto comprobamos cómo una determinada patología tiene manejo y costos diferentes sea que la E.P .S. a la que pertenece la paciente autorice o no autorice la Cirugía por Laparoscopia. 
Tomamos el caso de una paciente de 32 años que consultó por Dolor Pélvico Crónico (DCP) de ocho meses de evolución durante los cuales recibió múltiples tratamientos antibióticos y antiinflamatorios, y le fueron practicados numerosos exámenes de laboratorio y ecográficos. Esta paciente finalmente fue remitida a nuestra Unidad para una LAPAROSCOPIA DIAGNOSTICA. Después de examinar a la paciente solicitamos a la E.P.S.la autorización para realizar una LAPAROSCOPIA OPERATORIA por sospechar patología intraabdominal, pero se negó dicha posibilidad. Se realizó la LAPAROSCOPIA DIAGNOSTICA y se encontró un HIDROSALPINX IZQUIERDO GRADO II y un QUISTE ENDOMETRIOSICO DERECHO (4cm de diámetro).

Posteriormente, ante la lógica persistencia del D.P.C., se le realizó, un mes después, LAPAROTOMIA para practicar Salpingectomía Izquierda y Resección de Quiste de ovario derecho. En este caso llamamos a la Laparotomía: Laparotomía Diferida.

Se comparan costos causados por esta conducta (Laparoscopia y Laparotomía Diferida) contra los costos que se hubieran causado de autorizarse la LAPAROSCOPIA OPERATORIA solicitada inicialmente, en este caso Salpingectomía Izquierda más Cistectomía Derecha.

Para calcular los costos de los procedimientos se utilizó el Manual de Procedimientos y tarifás del Instituto de Seguros Sociales (I.S.S.), vigente en 1.999, por considerar que es uno de los más utilizados a nivel nacional, y más completo desde el punto de vista de la Endoscopia Ginecológica. Se puso especial énfasis en los artículos que, en este Manual de Tarifas, tienen indicaciones específicas para la facturación en cirugía laparoscópica (8).

Para los costos no estipulados estrictamente en el Manual, (costos variables dados principalmente por medicamentos y materiales que se facturan independientemente), se utilizaron los valores analizados en nuestro artículo de costos comparativos, ya publicado (1).

La estancia promedio para la cirugía por laparotomía se consideró de dos (2) días, realizada en una Institución de II Nivel de complejidad, con habitaciones bipersonales. Tanto la Laparoscopia Diagnóstica como la Laparoscopia Operatoria son cirugías ambulatorias, que no generan estancia hospitalaria.

La incapacidad causada para la Laparoscopia Diagnóstica y la Cirugía laparoscópica ambulatoria normalmente está entre 5 y 8 días y se tomó un promedio de siete (7) días para el caso analizado. La incapacidad promedio para la laparotomía está entre 20 y 30 días y se tomaron 25 días como promedio para la Cirugía por Laparotomía.

Para el cálculo del valor de la incapacidad laboral se utilizó el salario mínimo mensual vigente en Colombia, el cual es de \$ 236.460 (\$7.882 día).

\section{Resultados}

En la Tabla 1 se indican los procedimientos quirúrgicos que se analizarán desde el punto de vista de costos, con la vía, el código del Manual del I.S.S. y las Unidades de Valor Relativo (U. V .R.) que le corresponden.

En la Tabla 1 se pueden apreciar los costos totales que se hubieran causado si la patología de la paciente se hubiera resuelto, en el mismo acto quirúrgico, mediante Laparoscopia Operatoria. En este caso no se puede cobrar Laparoscopia Diagnóstica más Laparoscopia Operatoria, ya que la normatividad del Manual tarifario del I.S.S. así lo determina. El segundo procedimiento laparoscópico no se puede facturar por la misma razón (8).

En la Tabla 3 se incluyen los costos de la Laparoscopia Diagnóstica realizada y de los procedimientos de la Laparotomía Diferida practicada un mes después. El segundo procedimiento de la Laparotomía (Resección de Quiste de ovario derecho) se factura con el $60 \%$ para honorarios médicos y $50 \%$ para derechos de sala y materiales quirúrgicos (8).

En la Tabla 4 están resumidos los resultados totales de las tablas anteriores. También se incluye, con fines comparativos, el costo del procedimiento laparoscópico operatorio cuando se factura con la modalidad de Conjunto de Atención en Salud por Tarifa Integral ("Paquete") (8).

\section{Discusión}

Con el desarrollo y evolución de herramientas diagnósticas precisas, confiables, económicas y no invasivas (10-12), o incluso "menos invasivas" (13-14), las indicaciones de la laparoscopia diagnóstica sin posibilidad terapéutica no tienen razón de ser en la práctica ginecológica moderna.

Los resultados son evidentes. Analizando la información presentada en la Tabla 4 salta a la vista que ante la indicación de una laparoscopia diagnóstica si se le niega a la paciente la posibilidad de que se le realice, en el mismo acto quirúrgico, una laparoscopia operatoria, prácticamente se están DUPLICANDO los costos de la atención (97 a 124\%).

Las indicaciones para la laparoscopia ginecológica, hoy en día, pueden resumirse como diagnóstica o terapéutica. La diagnóstica puede ser urgente (dolor pélvico agudo, sospecha de perforación uterina), o electiva (Dolor pélvico crónico, infertilidad) (15). Como se puede apreciar, todas las indicaciones mencionadas en lalaparoscopia diagnóstica implican la posibilidad de tener que realizar procedimientos intraoperatorios para resolver la situación de la paciente (15-16).

El caso aquí presentado corresponde a una paciente de edad promedio con una sintomatología de Dolor Pélvico

Tabla 1

\begin{tabular}{|l|c|c|c|}
\hline \multicolumn{1}{|c|}{ PROCEDIMIENTO } & VIA & CODIGO & U.V.R. \\
\hline Laparoscopia Diagnóstica & & 18601 & 75 \\
Salpingectomía izquierda & Laparoscopia & 18914 & 120 \\
Cistectomía derecha & Laparoscopia & 18920 & 110 \\
Salpingectomía izquierda & Laparotomía & 11201 & 120 \\
Resección de Quiste o Tumor de Ovario & Laparotomía & 11106 & 100 \\
\hline
\end{tabular}


Crónico (PPC). Se tomó este caso en especial porque en nuestro medio el DCP es una causa muy frecuente de consulta ginecológica. En el Instituto Materno Infantil de Santafé de Bogotá lo reportan como el cuarto motivo de consulta en el área de consulta externa y como la indicación de las dos terceras partes de las laparoscopias realizadas en los últimos siete años (17-18). En nuestra unidad el DCP es el tercer motivo de consulta ginecológica y la principal indicación de laparoscopia diagnóstica o terapéutica. Además, es bien conocida la relación entre DCP y endometriosis, especialmente en estados avanzados (19-20), y la alta frecuencia de adherencias encontradas en laparoscopias realizadas por DCP (21).

Se sabe que entre un $61 \%$ de las pacientes a quienes se les realiza laparoscopia por DCP mostrarán anormalidades (principalmente endometriosis, adherencias, enfermedad pélvica inflamatoria, quistes de ovario y várices pélvicas), y un $78 \%$ de adolescentes con DCP mostrarán alguna patología (especialmente endometriosis) (22).

Tabla 2

\begin{tabular}{|c|c|c|}
\hline CONCEPTO & \multicolumn{2}{|c|}{$\begin{array}{l}\text { Salpingectomía izquierda } \\
\text { y Cistectomía derecha }\end{array}$} \\
\hline Consulta Preanestésica & $\$$ & 11,350 \\
\hline Consulta Prequirúrgica & $\$$ & 11,350 \\
\hline Honorarios Anestesia & $\$$ & 109,800 \\
\hline Honorarios Cirujano & $\$$ & 145,200 \\
\hline Honorarios Ayudante & $\$$ & 41,400 \\
\hline Derechos de Sala & $\$$ & 126,770 \\
\hline Drogas (promedio) & $\$$ & 31,575 \\
\hline Trocar desechable & & \\
\hline $1^{\text {a. }}$ Punción & $\$$ & 263,200 \\
\hline Material Quirúrgico & $\$$ & 113,520 \\
\hline Patología (Cod. 20201) & $\$$ & 26,450 \\
\hline Incapacidad & $\$$ & 55,174 \\
\hline Totales & $\$$ & 935,789 \\
\hline
\end{tabular}

En este sentido el caso descrito puede considerarse como frecuente y representativo del grupo de pacientes con indicación de laparoscopia diagnóstica, especialmente de pacientes con DPC.

Llama la atención el valor, para este caso, del Conjunto de laparoscopia operatoria de nivel avanzado (Tabla 4). Los costos de los conjuntos de cirugía laparoscópica, tanto de los manuales del ISS eomo del SOAT (8-9), promedian varios procedimientos $\mathrm{y}$ por consiguiente no dan lugar a cobros adicionales por patología bilateral. o por procedimientos múltiples. El uso de "paquetes" o conjuntos de cirugía ginecológica laparoscópica facilitan que ante una patología indeterminada, como por ejemplo DCP, el ginecólogo pueda optar por la conducta quirúrgica adecuada sin necesidad de realizar engorrosos trámites administrativos intra o postoperatorios. Además la E.P.S. puede controlar mejor el gasto, disminuye costos administrativos y prácticamente elimina la posibilidad de glosas en las cuentas presentadas, siempre y cuando se ajusten al "Paquete" autorizado.

Aunque los estudios de costos en cirugía por laparoscopia realizados en otros países son de utilidad (23-27), las características de la atención médica de cada lugar, y la utilización en Colombia de Manuales Tarifarios con especificaciones y limitantes solo aplicables en nuestras instituciones, no permiten extrapolar sus resultados.

Con el paso del tiempo en Colombia, al igual que en otros países, la difusión de la cirugía ginecológica por laparoscopia parece mostrar una tendencia progresiva en la disminución de costos $(1,27)$, cuando se realiza en condiciones adecuadas, y con personal experimentado y certificado (4-5).

Los beneficios para las pacientes (26), y la baja tasa de complicaciones y de morbi-mortalidad (28-30), hacen de la cirugía ginecológica laparoscópica una de las herramientas con mayor proyección e impacto en el futuro.

$\mathrm{Se}$ debe entonces continuar realizando estudios de costos en esta área, y en nuestro medio, con el fin de conseguir información confiable que permita evaluar

Tabla 3

\begin{tabular}{|c|c|c|c|c|c|c|c|c|}
\hline \multirow{2}{*}{$\begin{array}{l}\text { CONCEPTO } \\
\text { Consulta Preanestésica }\end{array}$} & \multicolumn{2}{|c|}{$\begin{array}{l}\text { Laparoscopia } \\
\text { diagnóstica }\end{array}$} & \multicolumn{2}{|c|}{$\begin{array}{l}\text { Salpingectomía } \\
\text { Izda. }\end{array}$} & \multicolumn{2}{|c|}{$\begin{array}{l}\text { Resec. Quiste } \\
\text { ovario der. }\end{array}$} & \multicolumn{2}{|c|}{ TOTALES } \\
\hline & $\$$ & 11,350 & $\$$ & 11,350 & & & $\$$ & 22,700 \\
\hline Consulta Prequirúrgica & $\$$ & 11,350 & $\$$ & 11,350 & & & $\$$ & 22,700 \\
\hline Honorarios Anestesia & $\$$ & 68,625 & $\$$ & 109,800 & $\$$ & 65,880 & $\$$ & 244,305 \\
\hline Honorarios Cirujano & $\$$ & 90,750 & $\$$ & 145,200 & $\$$ & 87,120 & $\$$ & 323,070 \\
\hline Honorarios Ayudante & $\$$ & 25,875 & $\$$ & 41,400 & $\$$ & 24,840 & $\$$ & 92,115 \\
\hline Derechos de Sala & $\$$ & 95,095 & $\$$ & 126,770 & $\$$ & 63,385 & $\$$ & 285,250 \\
\hline Drogas (promedio) & $\$$ & 31,575 & $\$$ & 147,520 & & & $\$$ & 179,095 \\
\hline Trocar desechable $1^{\mathrm{a}}$. Punción & $\$$ & 263,200 & & & & & $\$$ & 263,200 \\
\hline Material Quirúrgico & $\$$ & 76,720 & $\$$ & 113,520 & $\$$ & 56,760 & $\$$ & 247,000 \\
\hline Habitación dos días & & & & & & & & \\
\hline (Bipersonal Nivel II) & $\$$ & 111,380 & & & & & $\$$ & 111,380 \\
\hline Patología (Cod. 20201) & $\$$ & 26,450 & $\$$ & 26,450 & & & $\$$ & 52,900 \\
\hline Incapacidad & $\$$ & 55,174 & $\$$ & 197,050 & & & $\$$ & 252,224 \\
\hline VALOR TOTAL & $\$$ & 756,164 & $\$$ & $1,041,790$ & $\$$ & 297,985 & $\$$ & $2,095,939$ \\
\hline
\end{tabular}


Tabla 4

\begin{tabular}{|c|c|c|}
\hline PROCEDIMIENTO & \multicolumn{2}{|c|}{ VALOR TOTAL } \\
\hline $\begin{array}{l}\text { Laparoscopia Diagnóstica }+ \\
\text { Laparotomía Diferida } \\
\text { Conjunto Laparoscopia Operatoria }\end{array}$ & $\$$ & $2,095,939$ \\
\hline Nivel Avanzado (Cod. 40534) & $\$$ & $1,064,895$ \\
\hline Laparoscopia Operatoria & $\$$ & 935,789 \\
\hline
\end{tabular}

adecuadamente la variable costo-beneficio en la atención de la ginecología en Colombia.

\section{Conclusiones}

La práctica de la laparoscopia diagnóstica sin permitir la posibilidad de realizar, en el mismo acto quirúrgico, procedimientos operatorios, causa incrementos injustificados y excesivos en los costos de la atención de las pacientes, que en algunos casos puede llegar a ser de un 97 a un 124\%. Esto unido a la frecuencia de patología encontrada en pacientes con DCP implica que todas las laparoscopias diagnósticas realizadas a pacientes con DCP deban permitir realizar procedimientos intraoperatorios por laparoscopia. Esta posibilidad debe discutirse ampliamente con la paciente y se debe obtener consentimiento escrito antes de realizar cualquier procedimiento diagnóstico y/o operatorio.

La utilización de los Conjuntos de Atención en Salud por Tarifa Integral o "Paquetes" de cirugía ginecológica laparoscópica tiene ventajas por facilitar el control de gastos, el manejo administrativo y por disminuir la posibilidad de glosas en las cuentas presentadas.

Se justifica continuar realizando estudios de costos en el área de la cirugía ginecológica laparoscópica, en nuestro medio.

\section{BIBLIOGRAFIA}

1. Argüello R. Costos comparativos entre laparoscopia ginecológica ambulatoria y Cirugía ginecológica por laparotomía. Rev Colom Obstet y Ginecol; 1999; 50: 101-105.

2. Broussard T, Mckernan J, Laws H. Horizons in Endoscopic Surgery. En: Braverman M, Tawes R, eds. Surgical Technology lnternational II. 1a. ed. San Francisco, California. Editado por Surgical Technology lnternational 1993; 37-39.

3. Litynski G, Szabó Z.; Laparoscopic R/Evolution: Impetus to a New Understanding of Surgery; En: Szbó Z, Lwis J, Fantini G, Savalgi R, eds.; Surgical Technology international V.; 1a. ed. San Francisco, California; Universal Medical Press, Inc.: 1966; 95-100.

4. Argüello R. Entrenamiento y certificación en endoscopia ginecológica; Rev Colom Obstet y Ginecol; 1998; 49: 111-114.

5. Manual de Reglamentación en Endoscopia Ginecológica. Sociedad Colombiana de Obstetricia y Ginecología. Santafé de Bogotá: 1997.

6. Nava R, González E. Indicaciones, contraindicaciones y limitaciones de la cirugía laparoscópica. Ventajas y desventajas. En: Nava R, Molina A, eds. Endoscopia Quirúrgica Ginecológica. la. Ed. México: Mcgraw-Hill Interamericana Editores S.A.; 1997; 11-15.

7. Future Directions. En: Gomel V, Taylor P, eds. Diagnostic and Operative Gynecologic Laparoscopy. St. Louis, Missouri: MosbyYear Book, inc.; 1995; 310-312.

8. Instituto de Seguros Sociales. Acuerdo número 209 de 1999. Diario Oficial No. 43567, Abril 30, 1999.

9. Ministerio de Salud. Manual de Tarifas y Procedimientos. Decreto 2423 del 31 de Diciembre de 1996. (S.O.A.T.)

10. Torres J, Suso J, Perea E, et al. Utilización de un índice clínicoecográfico para la clasificación de los tumores de ovario: Rev Colom Obstet y Ginecol; 1998; 49; 141-144.

11. Recuento histórico y avances de la ecografía en obstetricia y ginecología. Controv. Ginec. Obst. 1998; 2(14): 15-25.

12. Kleinkauf H, Huneke B, Lindner C, Braendle W, Combining Bmode ultrasound with pulsed wave Doppler for the assessment of tubal patency: Hum. Reprod.; 1997; 12(11): 2457-60.

13. Nezhat C, Daniel J, Feste J, Palter S. Minilaparoscopia de consultorio: Controv. Ginec. Obst. 1997; 1(6): 33-37.

14. Palter S. Office microlaparoscopy under local anesthesia: Obstet Gynecol Clin North Am; 1999; 26(1): 109-20, vii.

15. Awwad J, Isaacson K. Indications and Contraindications of Laparoscopy. En: Cohen S, eds. Operative Laparoscopy and Hysteroscopy. 1a. Ed. New York, New York: Churchill Livingstone Inc.; 1996; 5-19.

16. Porpora M, Gomel V. The role of laparoscopy in the management of pelvic pain in women of reproductive age: Fertil Steril; 1997; 68 (5): 765-79.

17. Gómez P. Dolor Pélvico Crónico. En: Gómez P, Ruiz A, eds. Temas de interés en ginecología y obstetricia. la. Ed. Santafé de Bogotá:
Universidad Nacional de Colombia, Facultad de Medicina. Instituto Materno Infantil; 1998; 359-372.

18. Gómez P. Martín E. Clínica de Dolor Pélvico Crónico del Instituto Materno Infantil de Santafé de Bogotá D. C. Universidad Nacional de Colombia - Experiencia de nueve años; Controv. Ginec. Obst.; 1999; 4(19): 52-57.

19. Laparoscopic Treatment of Endometriosis. En. Nezhat C, Nezhat F, Luciano A, et al, eds. Operative Ginecologic Laparoscopy; la Ed: New York, New York; McGraw Hill, Inc.: 1995; 121-147.

20. Chapron C, Dubuisson JB. Laparoscopic treatment of deep endometriosis located on the uterosacral ligaments: Hum Reprod; 1996; 11(4): 868-73.

21. Steege J. Adhesions and pelvic pain. En: Steege J, Metzger D, Levy B, eds. Chronic Pelvic Pain. An Integrated Approach; Philadelphia, Pennsylvania; W. B. Saunders Company; 1998; 115-125.

22. Howard F. The role of Laparoscopy in Chronic Pelvic Pain: Promise and Pitfalls; Obstet Gynecol Surv; 1993; 48(6): 357-87.

23. Relative Value Units and Current Procedural terminology Codes. En: Hulka J, Reich H, eds. Textbook o Laparoscopy. 3rd. Ed. New York, New York: W. B. Saunders Company; 1998; 525-527.

24. Abascal A, Pérez A, Méndez F. Estudio de costo beneficio, costo eficiencia y costo financiero de la cirugía endoscópica. En: Nava R, Molina A, eds. Endoscopia Quirúrgica Ginecológica. 1a. Ed. México: McGraw-Hill Interamericana Editores S.A.; 1997; 58-60.

25. Johns A. Cost Effectiveness and Laparoscopic Surgery. En Adamson D, Martin D, eds. Endoscopic Management of Gynecologic Disease, Philadelphia, Pennsylvania: Lippincort-Raven Publishers; 1996; 415419.

26. Azziz R. Advantages and Disadvantages of Operative Endoscopy. En: Azziz R, Alvarez A, eds. Practical Manual of Operative Laparoscopy and Histeroscopy. Springer Verlag. New York, inc. 1992; 1-6.

27. Savalgi R. Economic Considerations in Laparoscopic Surgery: Disposable Versus Reusable Instruments. En: Szbó Z, Lwis J, Fantini G, Savalgi R, eds.; Surgical Technology international V.; 1a. ed. San Francisco, California; Universal Medical Press, Inc.: 1966; 109-112.

28. Querleu D, Chevallier L, Chapron C, Bruhat M. Complication of laparoscopy surgery. A French multicentre collaborative study. Gynaecol. Endosc. 1993; 2: 3-6.

29. Survey Data: Mortality and Morbidity. En Hulka J, Reich H, eds. Textbook of Laparoscopy. 3rd. Ed. New York, New York: W. B Saunders Company; 1998; 505-511.

30. Newton M. Complications of abdominal operations. En Newton M, Newton E, eds. Complications of Gynecologic and Obstetric Management. Philadelphia, Pennsylvania: W. B. Saunders Company; 1998; 143-174. 